
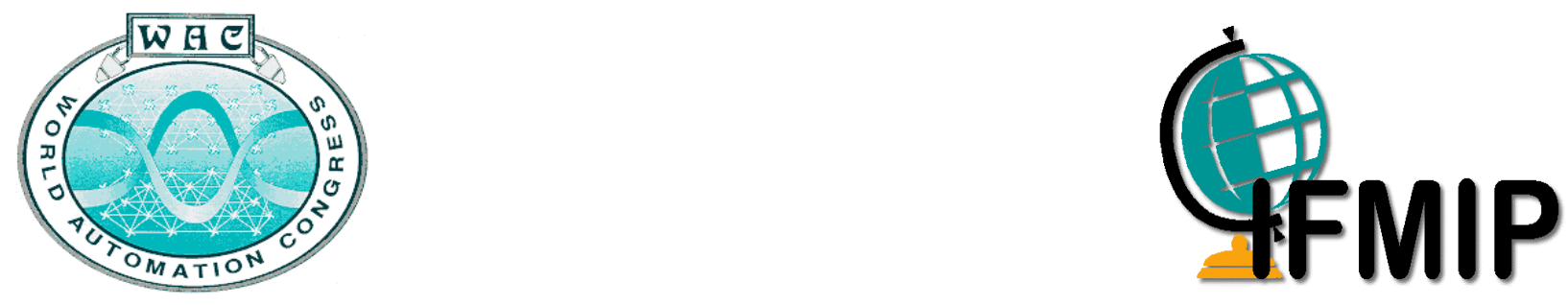

\title{
World Automation Congress
}

Third International Forum on Multimedia \& Image Processing

\author{
Orlando, Florida, USA \\ June 9-13, 2002
}

High-Sensitivity And Wide-Dynamic-Range Range Finder And Its Applications

Yusuke Oike, Makoto Ikeda, Kunihiro Asada 


\title{
HIGH-SENSITIVITY AND WIDE-DYNAMIC-RANGE RANGE FINDER AND ITS APPLICATIONS
}

\author{
YUSUKE OIKE MAKOTO IKEDA KUNIHIRO ASADA
}

\author{
Dept. of Electronic Engineering \\ VLSI Design and Education Center \\ University of Tokyo \\ 7-3-1 Hongo, Bunkyo-ku, Tokyo 113-8656, Japan
}

\begin{abstract}
We propose a high-sensitivity and wide-dynamic-range position sensor for range finders using logarithmic-response and correlation circuit. The 3-D measurement system using the proposed position sensor has advantages to applications, such as a walking robot and a recognition system on vehicles, which require both of availability in various backgrounds and safe light projection for human eyes. The position sensor with a $64 \times 64$ pixel array has been developed and successfully tested. We describe the sensitivity of position detection as SBR (Signal-to-Background Ratio). The minimum SBR of the sensor is $-14.4 \mathrm{~dB}$ lower than standard sensors. High sensitivity under $-10 \mathrm{~dB}$ SBR is realized in the dynamic range of $46 \mathrm{~dB}$ in terms of background illumination. Experimental results of position detection and 3-D measurement in a strong background illumination are also presented.
\end{abstract}

KEYWORD: 3-D measurement, position sensor, high sensitivity, Signal-to-Background Ratio(SBR), wide dynamic range, logarithmic response, correlation

\section{Introduction}

3-D measurement system has a wide variety of application fields such as robot vision, computer vision and position adjustment. In 3-D measurement system using triangulation-based light projection method, a sensor detects the position of the projected light on the sensor plane. Some applications, for example a walking robot and a recognition system on vehicles, require both of availability in various backgrounds and safe light projection for human eyes.

Standard imagers and most of the smart position sensors [1]-[7] detect positions of peak intensity on the sensor plane to acquire the position of the projected light. Therefore these sensors require strong light projection when a target object is placed in a non-ideal environment such as a strong background illumination. Color filters mounted on the sensors suppress the background illumination and realize a high sensitivity of position detection. Sunlight has, however, distributed waveslengths with strong intensity, so that color filters are not always enough for some applications. A highsensitivity position sensor with a capability of electronic suppression of background illumination is required in such situations.

Correlation technique [8] is one of the solutions to the problems. The correlation sensor can suppress the background illumination to obtain a high sensitivity. Its dynamic range is, however, limited by the linear difference circuit due to the voltage signal saturation. It is not applicable for a strong contrast image in outdoor environment. The position sensor with electronic shutter [9] can prevent the saturation for the problem of [8]. Its dynamic range is decided by the limit of the shutter interval and the extremely short shutter intervals decrease SNR. In addition, it is difficult to adjust the optimal shutter interval autonomously, especially in ununiform backgrounds. 
In this paper, we present a position sensor with $64 \times 64$ photo detectors using logarithmicresponse and correlation circuit for the 3-D measurement system. Wide-dynamic-range and highsensitivity position detection can be simultaneously realized without saturation. We show the performance of the sensor as well as the experimental results of position detection for 3-D measurement.

\section{Pixel Circuit}

Figure 1 illustrates the sensing scheme using logarithmic-response and correlation circuit. The photo detector receives the projected laser beam modulated by a pulse generator. The pixel consists of a photo detector with a logarithmic-response amplifier, a sample and hold circuit, a differential circuit, an analog multiplier for correlation and an integrator with a source follower output.

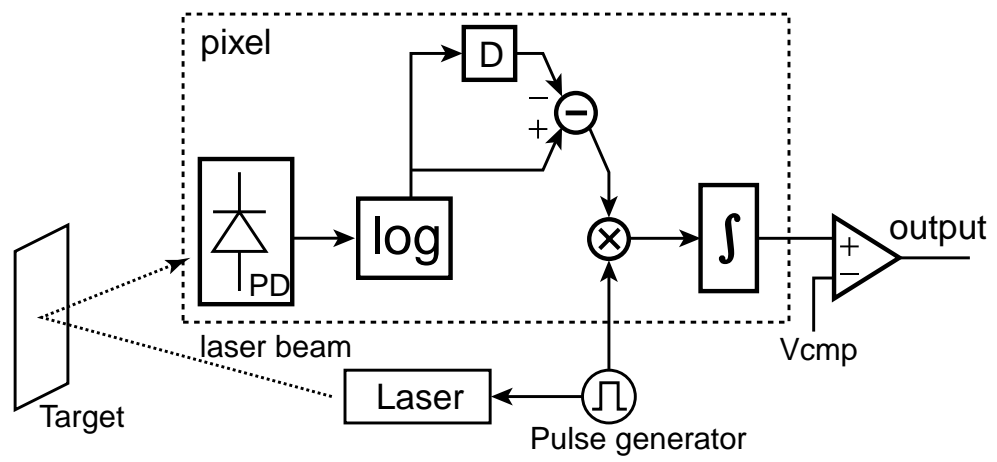

Figure 1: Sensing scheme of the proposed photo detector.

Figure 2 shows a schematic of the pixel. The logarithmic-response circuit realizes widedynamic-range photo detection. The photo current $I_{P D}(t)$ generates the voltage $V_{\text {sig }}(t)$ at the node sig as follows:

$$
V_{s i g}(t)=\alpha \log I_{P D}(t)
$$

Here $\alpha$ stands for the characteristics of the pre-amplifier. At the sample and hold circuit, the signal ref_sw synchronized with $2 f_{0}$ generates the voltage $V_{r e f}(t)$ at the node $r e f$ when the modulation frequency of the projected light to be detected is $f_{0}$. The differential voltage $\Delta V_{\text {sig }}(t)$ between $V_{\text {sig }}(t)$ and $V_{\text {ref }}(t)$ is multiplied by the external differential signal $\Delta V_{\text {sync }}(t)$ between $V_{\text {sync } 1}$ and $V_{\text {sync } 2}$ for correlation. $V_{\text {sync1 } 1}$ and $V_{\text {sync2 } 2}$ are synchronized with $f_{0}$. The output current $I_{\text {out }}(t)$ is integrated at the capacitance $C_{0}$ and results in the output voltage $V_{\text {out }}(t)$ as follows:

$$
\begin{gathered}
V_{\text {out }}(t)=\frac{1}{C_{0}} \int_{t-T}^{t} I_{\text {out }}(\tau) d \tau+V_{0} \\
I_{\text {out }}(t)=I_{b} \tanh \frac{\kappa \Delta V_{\text {sig }}(t)}{2} \tanh \frac{\kappa \Delta V_{\text {sync }}(t)}{2}
\end{gathered}
$$

where $T$ is frame interval and $\kappa$ is a gain factor of the multiplier. $V_{0}$ is an output bias voltage of the integrator. $V_{\text {out }}$ increases monotonously when the input signal has the frequency $f_{0}$. On the other hand, $V_{\text {out }}$ remains constant when the input signal is a constant background illumination. $V_{\text {out }}$ is compared to a reference voltage, $V_{c m p}$, to detect the laser signal position as follows:

$$
V_{\text {out }}(t) \geq V_{c m p}
$$




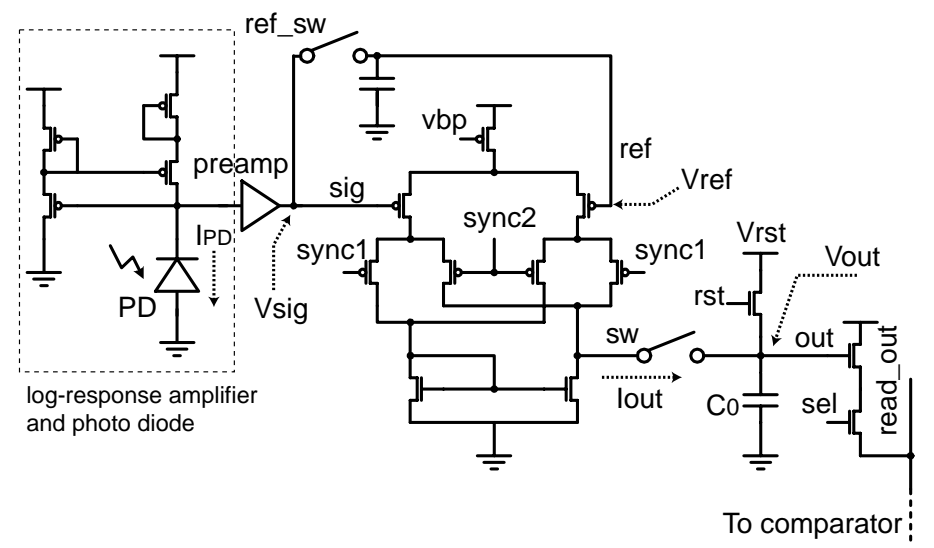

Figure 2: Schematic of the proposed photo detector.

\section{Chip Implementation}

We designed and fabricated a position sensor with a $64 \times 64$ proposed photo detector array in 0.5 $\mu m$ CMOS 3-Metal 1-Poly-Si process. Pixel size is $40 \mu m \times 40 \mu m$ and a photo diode occupies $18.05 \%$ in area. The photo diode is formed by an $\mathrm{n}^{+}$-diffusion in a p-substrate. The pixel has 24 transistors including transistor capacitances. Figure 3 shows a microphotograph and an array structure of the position sensor. It has row address decoders for select and reset, column-parallel source follower circuits, column-parallel comparators and an output multiplexer on a $4.8 \mathrm{~mm} \times 4.8$ $\mathrm{mm}$ die. The pixel values on a line are read out at a time and compared with the external reference signal $V_{c m p}$ in parallel. 64-bit outputs of comparators are multiplexed to 16-bit outputs.
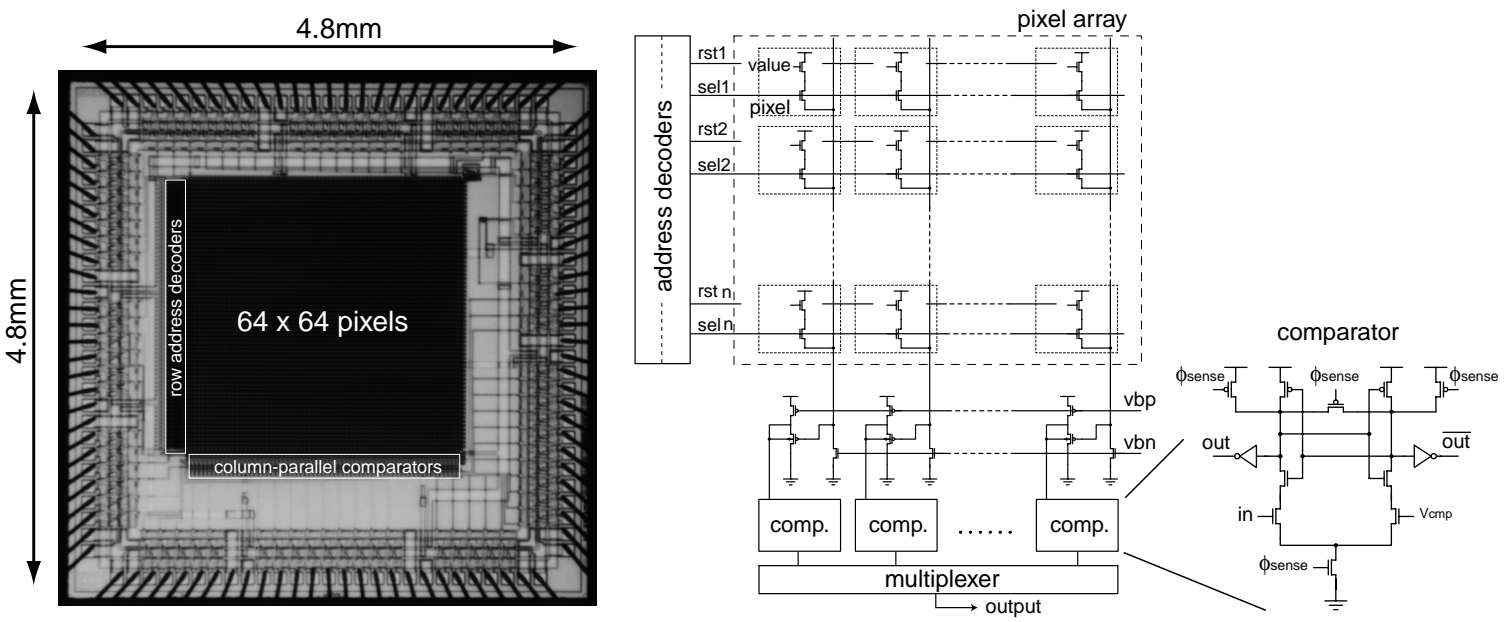

Figure 3: Microphotograph and an array structure of the position sensor.

\section{Performance Evaluation}

Figure 4 shows the output voltage, $V_{\text {out }}$, and suppression ratio at various input signal frequencies. The frequency $f_{0}$ for correlation is $1 \mathrm{kHz}$ and the frame interval $T$ is $10 \mathrm{~ms}$. The input signals with a frequency not equal to $f_{0}$ are suppressed. Especially, the suppression ratios of even-harmonics of $f_{0}$ are less than 0.05 . Therefore a set of frequencies such as $1 \mathrm{kHz}, 2 \mathrm{kHz}, 4 \mathrm{kHz}$ and $8 \mathrm{kHz}$ can be used in a multiple light projection system. Odd-harmonics of $f_{0}$ can't be fully suppressed due to the rectangular modulation. 


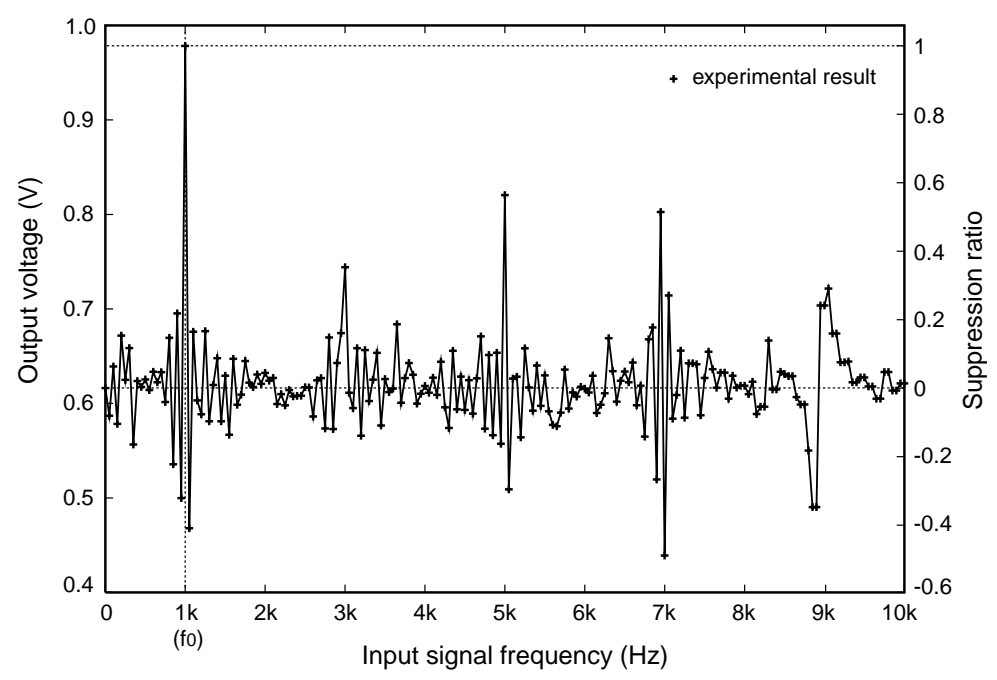

Figure 4: Output voltage and suppression ratio at various input signal frequencies.

The minimum laser intensity to be detected at each backgruond intensity is shown in Figure 5(a). In this measurement system, the modulation frequency is $1 \mathrm{kHz}$ and the frame interval is $10 \mathrm{~ms}$. The wavelength of projected laser beam is $635 \mathrm{~nm}$. This experimental result shows that the position sensor can suppress the background illumination. In addition, it shows that the highsensitivity position detection can be available without saturation in a wide range of background illumination. The minimum SBR (Signal-to-Background Ratio), which stands for the sensitivity of position detection, is $-14.4 \mathrm{~dB}$. High sensitivity under $-10 \mathrm{~dB}$ SBR is realized in the dynamic range of $46 \mathrm{~dB}$ in terms of background illumination. Figure 5(b) shows the performance of the conventional correlation sensor[8] in ideal operation. The voltage signals are saturated when the photo current increases in strong background illuminations, so that its dynamic range is limited. The conventional sensor realizes $-10 \mathrm{~dB}$ SBR at most in $13 \mathrm{~dB}$ of background illumination range. Note that position detection using a standard imager requires at least the same laser intensity as the background intensity (i.e. OdB SBR), especially in ununiform backgrounds. The minimum sensitivity of a standard imager is shown in Figure 5(c). Performance parameters of the proposed position sensor are summarized in Table 1.

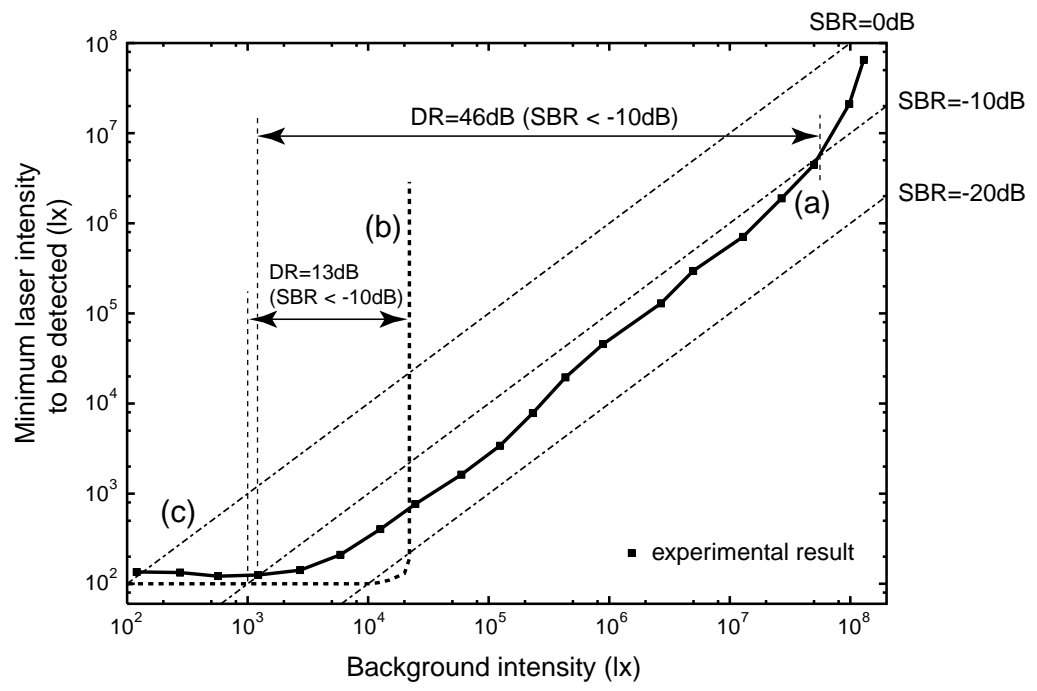

Figure 5: Dynamic range(DR) and signal-to-background ratio(SBR) of : (a) the fabricated position sensor, (b) the conventional correlation sensor[8], (c) a standard imager. 
Table 1: Performance parameters of the position sensor.

\begin{tabular}{ll}
\hline Power supply & $3.3 \mathrm{~V}$ \\
Minimum SBR & $-14.4 \mathrm{~dB}$ \\
DR (Dynamic Range) & $46 \mathrm{~dB}($ at $-10 \mathrm{~dB}$ SBR) \\
Depth resolution & $14.6 \mathrm{~mm}$ at $1418.0 \mathrm{~mm}$ \\
Chip power & $400 \mathrm{~mW}$ at $200 \mathrm{fps}$ \\
Max. frame rate & $2000 \mathrm{fps}$ (at $\left.f_{0}=10 \mathrm{kHz}\right)$ \\
\hline
\end{tabular}

\section{Application to Position Detection and 3-D Measurement}

Figure 6 illustrates the measurement system of the position detection in ununiform background illuminations. The laser intensity corresponds to $4 \mathrm{klx}$ and the maximum background illumination on the target object is about 60 klx. Figure 6(a-1) is acquired by a standard CCD camera and Figure $6(a-2)$ is acquired by the camera with the fabricated sensor when the laser beam is projected at Circle 1 on the board. In the same way, Figure 6(b-1) and (b-2) are acquired by each camera when the laser beam is projected at Circle 2. In Figure 6(a-1), the weak laser beam is projected at the area of the maximum background illumination, so that it is difficult to detect the laser beam position. In Figure 6(b-1), the weak laser beam is projected at another area. A standard CCD camera can't always detect only the laser beam position due to two or more peak intensities. On the other hand, the fabricated sensor can detect the laser beam position clearly such as Figure 6(a-2) and (b-2).

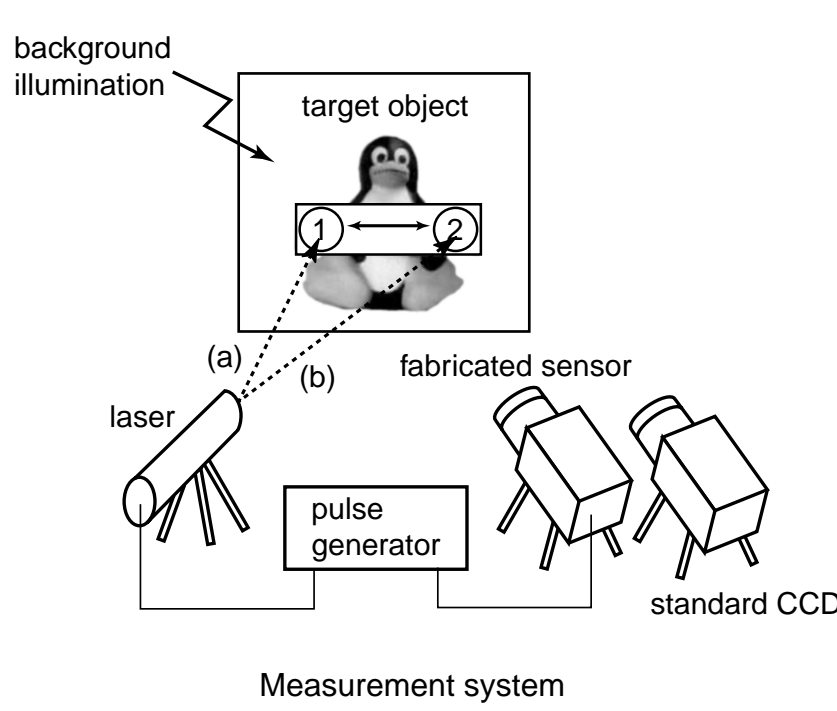

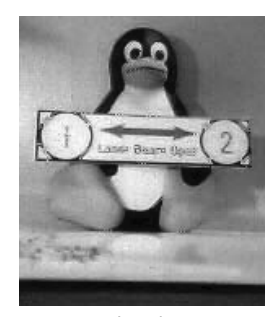

$(\mathrm{a}-1)$

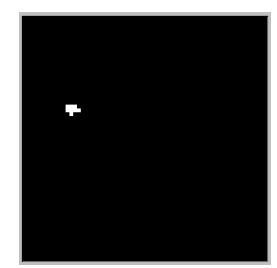

$(\mathrm{a}-2)$

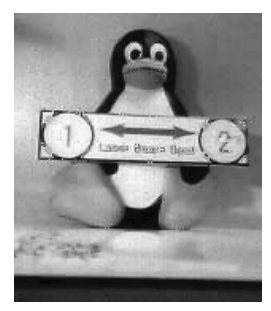

$(\mathrm{b}-1)$

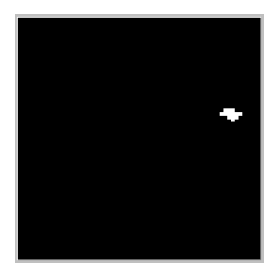

$(b-2)$

Acquired images

Figure 6: Measurement system and experimental results of the position detection in ununiform background illuminations.

Figure 7 illustrates the 3-D measurement system based on triangulation. The 3-D measurement system is composed of the fabricated sensor, a laser with mirrors and a PC with digital I/O boards. The sensor acquires the positions of the scanning laser spot. 3-D range map can be calculated from the positions of the projected laser beam on the acquired images and both positions of the sensor and the projected light source. The maximum error of measured range is $14.6 \mathrm{~mm}$, which corresponds to about $1 \%$. A sphere-shaped target object and a reproduced wire frame are shown in Figure 7. In this measurement, maximum background illumination on the target object is $28 \mathrm{klx}$ and the laser intensity corresponds to $1.6 \mathrm{klx}$. 

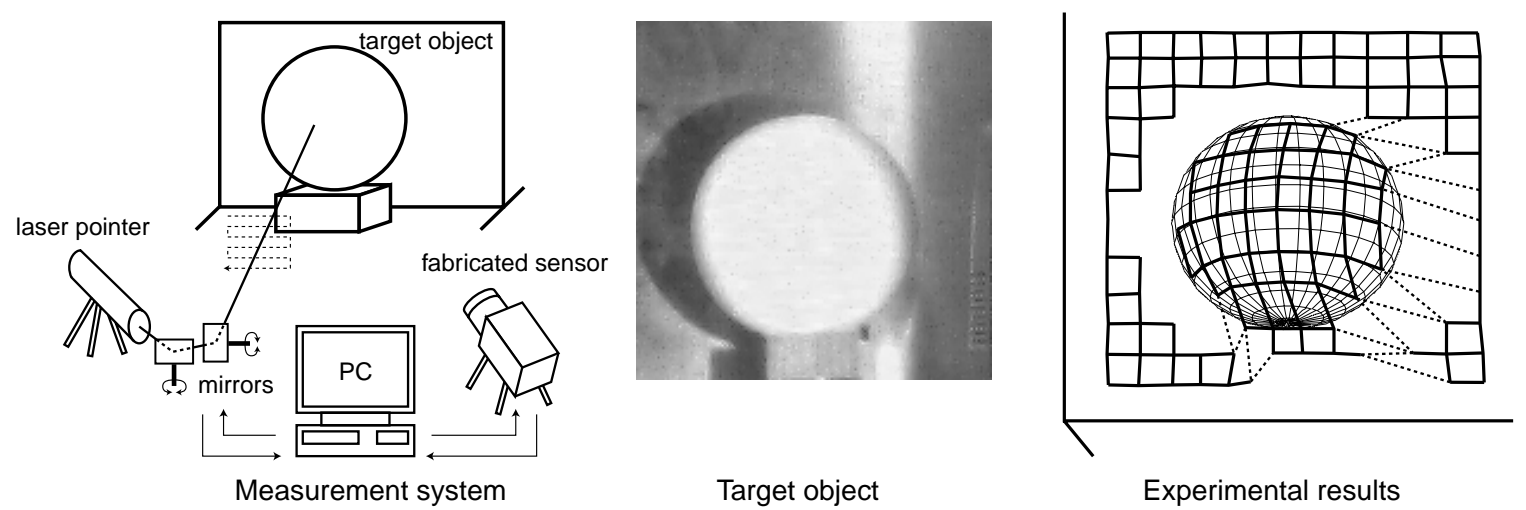

Figure 7: Block diagram of 3-D measurement system and a reproduced wire frame.

\section{Conclusion}

A high-sensitivity and wide-dynamic-range position sensor for range finders using logarithmicresponse and correlation circuit has been developed in $0.5 \mu \mathrm{m}$ CMOS process and successfully tested. The fabricated sensor has a $64 \times 64$ pixel array. The minimum SBR of the sensor is -14.4 $\mathrm{dB}$ less than standard imagers. High sensitivity under $-10 \mathrm{~dB}$ SBR is realized in the dynamic range of $46 \mathrm{~dB}$ of background illumination. A multiple light projection system is also available using a set of even-harmonics frequencies. We showed the experimental results of position detection for 3-D measurement system in ununiform background illuminations. The proposed sensor has advantages to applications which require both of availability in various backgrounds and safe light projection for human eyes.

\section{Acknowledgment}

This VLSI chip was fabricated in the chip fabrication program of VLSI Design and Education Center (VDEC), the University of Tokyo with the collaboration by Rohm Corporation, Toppan Printing Corporation, Hitachi Hokkai Semiconductor Ltd. and Dai Nippon Printing Corporation.

\section{References}

[1] A. Gruss, L. R. Carley and T. Kanade, "Integrated Sensor and Range-Finding Analog Signal Processor," IEEE J. of Solid-State Circuits, Vol 26, pp.184-191, 1991.

[2] M. Baba, T. Konishi, Y. Hori and K. Otani, "A New Position Sensor for High-Speed Measurement of Multiple Points," IEEE Proc. of Instrumentation and Measurement Technology Conference, pp.642-647, 1996.

[3] M. de Bakker, P. W. Verbeek, E. Nieuwkoop and G. K. Steenvoorden, "A Smart Range Image Sensor," Proc. of European Solid-State Circuits Conference, pp.208-211, 1998.

[4] V. Brajovic and T. Kanade, "Computational Sensor for Visual Tracking with Attention," IEEE J. of Solid-State Circuits, Vol. 33, pp.1199-1207, 1998.

[5] T. Nezuka, M. Hoshino, M. Ikeda and K. Asada, "A Smart Position Sensor for 3-D Measurement," Proc. of Asia South-Pacific Design Automation Conference, pp.21-22, 2001.

[6] S. Yoshimura, T. Sugiyama, K. Yonemoto and K. Ueda, "A 48kframe/s CMOS Image Sensor for Realtime 3-D Sensing and Motion Detection," ISSCC Dig. of Tech. Papers, pp.94-95, 2001.

[7] V. Brajovic, K. Mori and N. Jankovic, "100frames/s CMOS Range Image Sensor," ISSCC Dig. of Tech. Papers, pp.256-257, 2001.

[8] A. Kimachi and S. Ando, "Time-Domain Correlation Image Sensor: First CMOS Realization and Evaluation," Transducers '99, pp.958-961, 1999.

[9] R. Jeremias, W. Brockherde, G. Doemens, B. Hosticka, L. Listl and P. Mengel, "A CMOS Photosensor Array for 3D Imaging Using Pulsed Laser,” ISSCC Dig. of Tech. Papers, pp.252-253, 2001. 